\title{
Kernos
}

Revue internationale et pluridisciplinaire de religion grecque antique

$11 \mid 1998$

Varia

\section{Le sacrifice grec entre texte et image. À propos de l'ouvrage de F.T. VAN STRATEN, Hierà kalá}

\section{Pierre Bonnechere}

\section{OpenEdition \\ Journals}

\section{Édition électronique}

URL : http://journals.openedition.org/kernos/1234

DOI : 10.4000/kernos. 1234

ISSN : 2034-7871

\section{Éditeur}

Centre international d'étude de la religion grecque antique

Édition imprimée

Date de publication : 1 janvier 1998

ISSN : 0776-3824

\section{Référence électronique}

Pierre Bonnechere, «Le sacrifice grec entre texte et image. À propos de l'ouvrage de F.T. van STRATEN, Hierà kalá », Kernos [En ligne], 11 | 1998, mis en ligne le 21 avril 2011, consulté le 11 mars 2021. URL: http://journals.openedition.org/kernos/1234 ; DOl : https://doi.org/10.4000/kernos.1234 
Kernos, 11 (1998), p. 377-438.

\section{Revue des Livies}

\section{Compte rendu critique}

\section{Le sacrifice grec entre texte et image}

À propos de l'ouvrage de F.T. van STrRa'Ten, Hierà kalá ${ }^{1}$

par Pierre Bonnechere (Université de Montréal)

\section{L'iconographie du sacrifice animal}

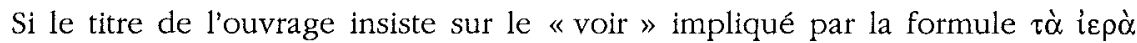

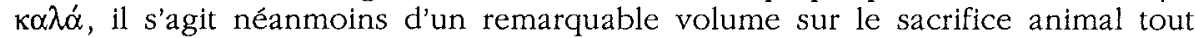
court. Aucune synthèse de cette qualité n'avait vu le jour depuis les ouvrages fondamentaux de Paul Stengel et de Samson Eitrem, datés respectivement de 1910 et $1915^{2}$, et la difficulté, voire l'aridité de ce sujet, sur lequel se fondent pourtant les théories des grandes écoles de pensée en histoire de la religion grecque depuis une trentaine d'années ${ }^{3}, n^{\prime} y$ est certes pas pour rien. Remarquablement informé, l'A. n'a cependant pas la prétention d'oblitérer les écrits de ses prédécesseurs, mais bien de les compléter avantageusement, en offrant un regard différent à partir de sources souvent négligées.

Le premier chapitre traite de méthode, énonçant des vérités simples si fréquemment oubliées : les figurations sur vases, qui datent des $\mathrm{VI}^{\mathrm{e}}$ et $\mathrm{v}^{\mathrm{e}}$ siècles, offrent d'importantes différences avec les reliefs des $\mathrm{v}^{\mathrm{e}}$ et $\mathrm{IV}$, sans que cela soit imputable à autre chose qu'à une question de type de matériel, rendant difficile toute comparaison conclusive entre les deux groupes. Chacun de ceux-ci, en outre, demeure sujet aux variations artistiques que l'on a trop souvent tendance à traduire en termes d'évolution cultuelle là où la seule évolution est celle de la mode. Quant à la répartition géographique de la documentation, elle se restreint à Athènes (avec quelques renseignements extérieurs, essentiellement corinthiens).

F. van Straten est un partisan de la méthode "What you see is what it is "; le «you » se rapporte évidemment à un Grec de l'Antiquité, allusion directe à la

1 F.T. van Straten, Hierà kalá. Images of Animal Sacrifice in Archaic and Classical Greece, Leiden-New York-Köln, E.J. Brill, 1995, 374 p., 168 fig. hors-texte (Religions in the Graeco-Roman World, 127). ISBN : 90-04-10292-2.

2 P. STENGEL, Opferbräuche der Griecben, Leipzig-Berlin, 1910 [réimpr. 1970]; S. ErTrem, Opferritus und Voropfer der Griecben und Römer, Kristiania (Oslo), 1915 [réimpr. 1977] (Videnskapsselskapets Skrifter. II. Hist.-Filos. Klasse, 1914, 1).

3 La remarquable synthèse offerte par J. RuDHARDT, O. REvERDin (éds), Le sacrifice dans l'Antiquité, Genève, 1981 (Entretiens sur l'Antiquité classique, 27) peut encore servir de base à cette assertion. 
conceptualisation grecque face à l'immolation des animaux; l'A' insiste sur le caractère à la fois nécessaire et illusoire d'une telle démarche (p. 5-9), selon une option méthodologique qui, à l'heure de l'iconologie-reine, est exemplaire. Je ne veux certes pas dire que cette dernière se berce de fausses certitudes, mais à la base de tout travail d'interprétation doit se trouver l'étape capitale du relevé méticuleux des témoignages. L'étude de ceux-ci selon des critères extérieurs à leur signification cachée mènera à des conclusions d'ordre externe qui conditionneront les hypothèses avancées quant à la signification profonde, iconologique au sens strict. Cette étape n'avait jamais été réalisée. Et l'A. a le don tout particulier de remettre en cause les évidences trompeuses.

Ainsi préconise-t-il l'établissement de séries iconographiques groupées autour de figurations de même type, et de n'adopter d'interprétation qu'au cas où cette dernière peut se justifier pour chacune des figurations de chaque série. Il s'interroge encore à propos de l'usage de l'objet qui porte la représentation, et adopte, pour les recoupements littéraires, une attitude de grande prudence par le biais de l'établissement d'importants dossiers comparatifs, afin d'éviter les pièges d'attestations faussées par leur caractère «artistique ». Par mesure de sécurité, toutes les données statistiques excluront les représentations mythiques, elles aussi reprises. Les chapitres 2 à 4 traitent respectivement des préparatifs de la mise à mort (p. 13-102), de l'immolation elle-même (p. 103-114) et enfin des actes succédant à la mise à mort (p. 115-160), selon la distinction de Walter Burkert. Le chapitre le plus attendu est le cinquième, qui met en perspective les trois précédents et synthétise les acquis de la démarche (p. 161-192). Viennent à la fin deux catalogues presque exhaustifs, remarquables et uniformisés, l'un consacré aux figurations sur vases (431 au total), le second aux reliefs votifs (243 au total, représentant du reste une minorité parmi le nombre total de reliefs votifs conservés). Chaque pièce est datée et décrite, accompagnée de sa bibliographie essentielle.

Les commentaires qui suivent s'inscrivent en pleine continuité avec le travail de van Straten, et soulignent simplement quelques points de désaccord audedans d'une adhésion très profonde à l'ensemble du livre.

Phase un : Pre-Kill. On croyait bien établie la relation entre la procession présentée sur une série de vases attiques et la procession des Panathénées, mais l'on s'aperçoit vite qu'il est bien plus vraisemblable que ces images n'aient fait qu'indiquer l'idée d'un sacrifice important en l'honneur de la déesse (p. 14-30). C'est d'ailleurs la conclusion générale à propos des processions figurées, à laquelle n'échappe pas d'ailleurs la série des vases sur les Diipolies (p. 51-52): l'artiste tient à donner une impression générale de fête plus que de se référer à un festival en particulier ${ }^{4}$. Un intéressant développement concerne l'usage simultané du panier qui contient la $\mu \alpha \dot{\alpha} \chi \alpha \iota \rho \alpha$ et du récipient qui renferme l'eau lustrale, la $\chi \dot{p} \rho \vee \imath \psi(p .31-49)$ : depuis Stengel, c'est la première tentative concluante de décrire ce moment du rituel avec toute la précision requise. La seule chose pour laquelle je prendrai l'A. à partie est l'apparente certitude de la dissimulation du couteau sacrificiel dans le kavỗv depuis le début de la procession (p. 32-33 et 4748). Les textes traditionnellement allégués sont Aristophane (Paix, 948) et ses scholiastes (ad loc.), mais le poète est très allusif, tandis que ses commentateurs

4 Cette conclusion rejoint, pour un sujet différent mais qui touche à l'intention des artistes et à l'attente du public, celle de K. Schauenburg (Zur Grabsymbolik apuliscber Vasen, in JDAI, 104 [1989], p. 19-60) pour les vases funéraires apuliens, où l'idée de salut semble très nettement primer sur l'exactitude du mythe représenté. 
semblent s'inspirer davantage du texte qu'ils glosent que d'informations indépendantes. Au reste, dans la Paix, le sacrifice ne comporte pas de procession, Trygée amenant au lieu du sacrifice les ustensiles et la victime au fur et à mesure qu'il les trouve'. Euripide, dans l'Iphigénie à Aulis, semble d'ailleurs indiquer la possibilité du contraire ${ }^{6}$. Cette prise de position ébranle quelque peu l'image que l'on se fait de la violence sacrificielle, dissimulée dans le panier d'une "candide canéphore ", mais ce n'est pas le lieu de s'attarder en détail à ce problème ${ }^{7}$.

La représentation, sur les reliefs votifs, du porteur de кฉvov̂v est digne d'intérêt, à deux égards : d'abord celui-ci ne tient que rarement la $\chi \chi^{\prime} p v i \psi$ (une seule exception, non assurée, en R 8 , ill. 59), sans que cette différence par rapport aux figurations sur vases et aux textes classiques puisse être expliquée de façon convaincante. Ensuite, la taille du porteur de la corbeille est souvent inférieure, parfois de moitié, à celle des autres personnes qui viennent honorer le dieu. Selon l'A. (p. 60-61 et 65-67), cette particularité n'aurait pas de rapport avec l'âge du personnage, mais bien avec son degré d'importance ${ }^{8}$ (au même titre que la porteuse de кí $\sigma \tau \eta$ dont seul l'objet qu'elle porte est essentiel ${ }^{9}$ ). Sans doute est-ce vrai en certains cas, notamment dans le relief $\mathrm{R} 75 \mathrm{bis}$ (ill. 88$)^{10}$, où le porteur tient en outre, exceptionnellement, le couteau : cet acte évidemment ne pourrait pas être celui d'un enfant; à la limite, par contre, il pourrait être celui d'un éphèbe ${ }^{11}$. L'on ne saurait cependant toujours s'en tenir à cette règle, car en bien d'autres cas, force est de constater, me semble-t-il, que les traits enfantins de ces personnages plaident à tout le moins en faveur d'un âge moins élevé que celui des autres participants (cas particulièrement clair en R 67, ill. 81) ${ }^{12}$. Au demeurant, le porteur de kavov̂v, qui sur les reliefs guide en même temps la victime, est

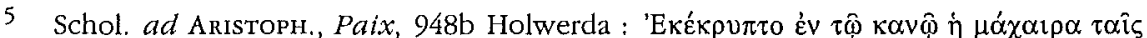

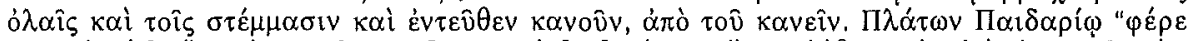

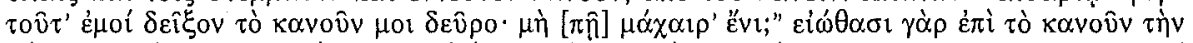

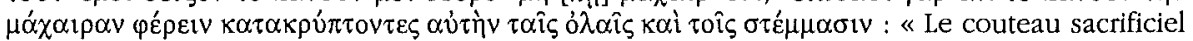

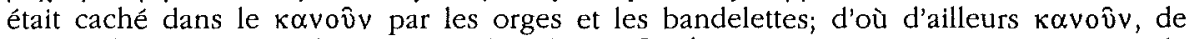

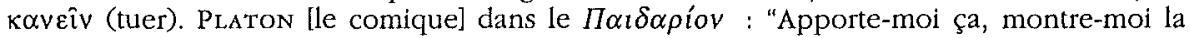
corbeille, vite fait : n'y a-t-il pas de couteau sacrificiel ?". On avait donc coutume de

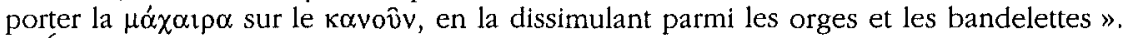

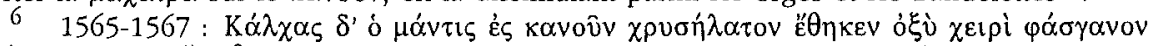

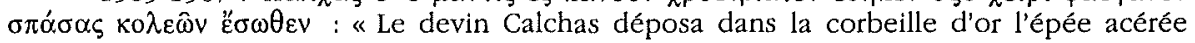
qu'il avait tirée du fourreau ».

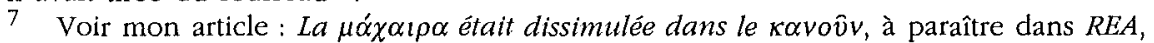
101 (1999)

8 Pourrait-on dès lors faire la même remarque en ce qui concerne la canéphore, souvent figurée plus petite, de façon à ce que le panier qu'elle porte sur la tête s'aligne au niveau de la tête des autres participants à la procession? Il n'empêche que les canéphores sont des jeunes filles, ayant certes connu leur développement physique et sans doute nubiles, mais dont l'âge devait tourner aux alentours de 16 ans seulement. On ne pourrait justifier non plus une importance inférieure allouée aux canéphores quand on sait que toutes les sources s'entendent pour faire de la canéphorie un acte honorable tant pour la personne aristocratique que pour la famille dont elle est issue.

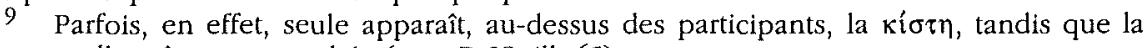
porteuse elle-même est occultée (ex. : R 28, ill. 65).

10 Lamia, AE 1041 (ca 300 av. J.-C.).

11 Souvent le porteur du panier semble être un éphèbe : par exemple R 106, ill. 102; R 68 (?), ill. 82; R 96, ill. 95; R 111, ill. 100; R 126, ill. 103; R 149, ill. 107 et R 188, ill. 105, où les victimes sont également miniatures (faudrait-il sinon leur supposer aussi une importance moindre?).

12 Voir aussi la pinax de Pitsá, ill. 56. 
toujours, quand il est figuré un peu plus âgé, imberbe, signe d'une classe d'âge qui au maximum peut être celle des éphèbes ${ }^{13}$. En cela, les reliefs me semblent corroborer les peintures sur vases, où ce rôle est toujours dévolu à un éphèbe, ou à un jeune esclave, mais en tous les cas à une personne imberbe. À propos des bergers portant un agneau ou un jeune animal sur leurs épaules (p. 55-56), on ne rejettera aucunement leurs relations avec Hermès criophore ou le monde pastoral, ni avec le sacrifice auquel ils pourraient être destinés, mais il est probable qu'il faille y voir en partie aussi, à l'instar de statuettes de Kato Symè, une agrégation à la vie d'adulte, sanctionnant une période de marge vécue avec un homme mûr ${ }^{14}$. Enfin, l'A. aborde la question du consentement de la victime (p. 100-102), qu'il considère comme une formalité automatique et très marginale dans l'accomplissement du rite sacrificiel. Toutefois, l'absence d'intérêt invoquée, de la part des artisans antiques, est difficilement utilisable pour servir de base à un tel raisonnement, sans compter qu'elle me semble heurter sur ce point de nombreuses allusions textuelles à cette opération.

Phase deux : The Killing. Après ses devanciers, van Straten constate l'aporie de documents figurés sur la mise à mort : deux reliefs (R 75bis, ill. 88, et R 225, ill. 109) et neuf vases, avec au total, sur onze représentations, deux seulement qui montrent l'acte de trancher la gorge, si l'on excepte l'amphore tyrrhénienne qui figure l'égorgement de Polyxène ${ }^{15}$.

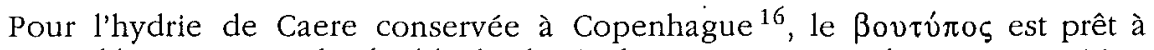
frapper: l'homme tient la double hache à deux mains, soit dans une position reliée au geste de porter le coup fatal. Étant donné la précision du peintre dans le rendu des attitudes, il est peu vraisemblable que l'artiste ait figuré le moment où la hache s'abat sur la victime, car le choc aurait lieu sur les cornes tandis que, d'autre part, la position de la jambe gauche de l'officiant, fléchie, ne permettrait pas à l'individu de frapper avec toute la force voulue (p. 107-108). Je pense plus vraisemblable que l'on se situe au moment où l'homme entame le mouvement du lever de la hache, à deux mains. D'autre part, le fait que l'assistant semble, outre le $\sigma \varphi \alpha \gamma \varepsilon i o v$, tenir aussi la $\mu \alpha \alpha \chi \alpha, ı \alpha$, ne semble pas impliquer un chevauche-

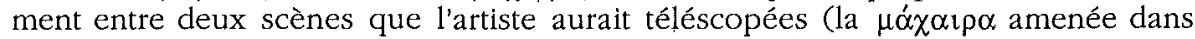

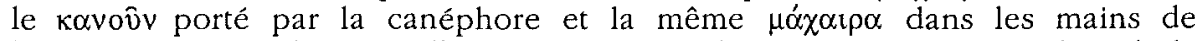
l'assistant), puisqu'il n'est nullement assuré que le couteau fut apporté lors de la

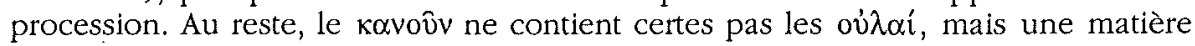
blanche indéterminée et bombée.

Phase trois : Post-Kill. Le chapitre consacré à la part des dieux est un modèle de méthode ("Curly things and bits on spits"): on y discute des os des cuisses, de parcelles de chair, de la queue (éventuellement avec le sacrum voire quelques vertèbres lombaires), la vésicule biliaire et une part de $\sigma \pi \lambda \alpha \dot{v} \chi v \alpha \alpha$ - on ignore comment cette dernière était offerte à la divinité (brûlée ou déposée dans les mains ou sur les genoux de la statue, ou encore posée dans la phiale de celleci). L'A. rapproche remarquablement le texte de la Paix d'Aristophane et les

13 Par exemple R 8, ill. 59; R 23, ill. 63; R 28, ill. 65; R 33, ill. 58; R 37, ill. 72; R 39, ill. 73; R 55, ill. 77; R 73, ill. 57; R 74, ill. 86; R 75, ill. 87; R 78, ill. 89; R 90, ill. 93. Une exception en $\mathrm{R} 92$, ill. 94, mais encore faut-il remarquer que l'homme qui guide les animaux ne tient pas le panier.

14 Voir en dernier lieu N. Marinatos, Minoan Religion. Ritual, Image, and Symbol, Columbia (South Carolina), 1993, p. 123 et notes p. 270.

15 Londres, $B M, 97.7-27.2$ : van Straten, Hierà kalá, V 422 et fig. 118.

$16 N M, 13567$ (V 120, ill. 114). 
représentations figurées, montrant la canonicité du schéma : \{brûler la part des

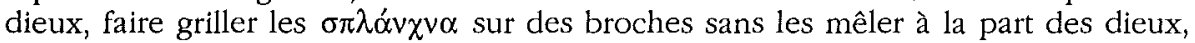
faire une libation et prier\}, et ce depuis l'époque archaïque (p. 118-141).

L'assimilation de petites boules, souvent rendues en rouge par les peintres et qu'un des assistants tient en main lors de cette troisième phase (vases décrits $p$. 139-141) avec les $\theta v \lambda \hat{\eta} \mu \alpha \tau \alpha$, boulettes de farine d'orge que l'on trempait de vin et d'huile d'olives et que l'on brûlait en complément au sacrifice, est pleine de bon sens (p. 141-144). Elle est de loin meilleure que l'hypothèse selon laquelle il se serait agi de grains d'orge, par trop rattachés aux préliminaires du sacrifice ${ }^{17}$.

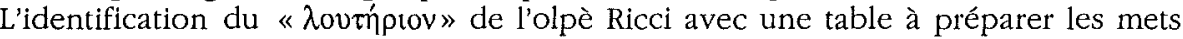
se signale également par son efficacité (p. 149-150).

Van Straten passe alors en revue les résultats acquis et met en perspective les trois temps du sacrifice. L'analyse qu'il propose du kovov̂v fera date (p. 162-164), On avait coutume, depuis la bonne synthèse de $J$. Schelp ${ }^{18}$ et déjà auparavant, de considérer que l'unique usage de la corbeille était de transporter, lors de la procession, les grains d'orge, une bandelette et le couteau sacrificiel. L'A. a montré, preuves à l'appui, bien d'autres utilisations de la corbeille, qui servait en

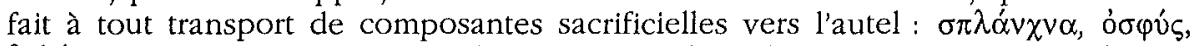
$\theta 0 \lambda \eta ́$ $\mu \alpha \tau \alpha$, gâteaux, récipients (huile et vin ?) et bien entendu grains d'orge. Poursuivant les conclusions d'un article d'A.D. Nock ${ }^{19}$, van Straten confirme ensuite par l'image que la distinction traditionnelle ${ }^{20}$ entre isacrifice olympien-

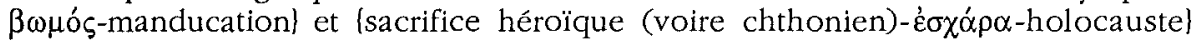
relève de l'érudition déficiente des scholiastes: plusieurs illustrations montrent en effet un sacrifice accompli sur un autel bas, dans la phase de rôtissage des $\sigma \pi \lambda \alpha v^{\prime} \chi v \alpha$, tandis que les reliefs voués à des héros, montrent très souvent des autels de type $\beta \omega \mu o^{21}$ (p. 165-167). Les remarques assez tranchées à propos du feu bouté sur l'autel laissent peut-être la place pour quelque hésitation (p. 168): certes les artisans ont souvent figuré de petites flammes pour indiquer le foyer en début de procédure sacrificielle, et de grands feux pour la phase de rôtissage des entrailles et de consomption de la part des dieux, mais il subsiste des exceptions trop nombreuses pour en tirer une règle dans la figuration antique des sacrifices $^{22}$. En ce qui concerne la détermination des tâches entre "masters and

17 Peut-être pourrait-on reconnaître ces boulettes d'avoine ou d'orge dans le kavov̂v d'une canéphore en pleine procession, sur une petite amphore corinthienne du début du

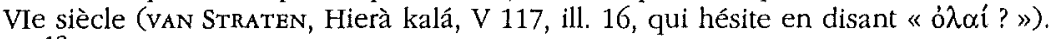

18 J. Schelp, Das kanoun. Der griechische Opferkorb, Wurzbourg, 1975 (Beiträge zur Arcbäologie, 8).

19 A.D. Nock, The Cult of the Heroes, in HThR, 37 (1944), p. 141-174.

20 Distinction qui a la vie longue : voir par exemple F. RoBer $\mathrm{T}$, La religion grecque, Paris, 1981 (Que sais-je?, 1969), p. 22-29.

21 À cet égard, la description que l'A. donne d'une oinochoé de la fin du ve siècle et représentant un « sacrifice à Héraclès » (p. 158) est à la limite de tomber dans ce travers justement dénoncé.

22 Seuls comptent ici les vases, puisque les reliefs comportaient peut-être un feu mais peint, et donc aujourd'hui disparu : V 21, ill. 4; V 50, ill. 5; V 52, ill. 24; V 127, ill. 30; V 136, ill. $34 \ldots$ L'hypothèse de J.-L. Durand, selon laquelle un autel est implicitement « virtuellement igné dans cette position iconique » est attractive, mais elle ne peut être acceptée que du print de vue logique (Images pour un autel, in R. Étienne, M.-Th. Le Dinahet 
servants » (p. 168-170), par ailleurs très utilement et clairement établie, on aurait bien voulu obtenir quelques précisions sur les femmes qui sont montrées en train de sacrifier (V 149, fig. 111, contexte dionysiaque et V 142, contexte " érotique "), de même que sur la signification des différences d'âge, souvent clairement soulignées.

Le choix de la victime fait l'objet d'une étude serrée (p. 170-186), qui relativise toute tentative d'établir des théories dogmatiques à partir des sources dont nous disposons, et la démonstration en vaut la chandelle! Se fondant sur les sources attiques, van Straten arrive à ces conclusions ${ }^{23}$ :

- Des calendriers, ces sources épigraphiques privilégiées ${ }^{24}$, il appert que les bovins constituent $10 \%$ des animaux sacrifiés, pour $60 \%$ aux ovins et $20 \%$ aux porcins.

- Sur les vases attiques, les bovins passent à $60 \%$, tandis que les deux autres catégories plafonnent peu en-dessous des $15 \%$.

- Sur les reliefs classiques, grecs cette fois, les bovidés totalisent à peine plus de $10 \%$, pour $35 \%$ aux ovins, alors que les porcins montent à $50 \%$.

En dépit des querelles possibles quant à la validité des échantillons statistiques, d'ailleurs exposées en long et en large ${ }^{25}$, il n'en reste pas moins étonnant, comme le dit l'A., que trois classes de documents censés présenter la même réalité divergent à ce point! C'est que chaque classe de documents trahit un «type de représentation » différent, et nécessite dès lors une explication qui lui est propre, tout amalgame débouchant sur des conclusions inacceptables.

Par la différenciation précise de la motivation et de la nature des sacrifices, l'A. tente d'expliquer ces différences, et y parvient avec un degré de certitude très raisonnable: les calendriers indiquent des fêtes publiques mais locales qui contrastent avec les fêtes publiques athéniennes, tandis que les reliefs sont le fait de particuliers ou de familles, soit ressortissant au domaine privé. Les vases, de leur côté, sont décorés pour appâter le client et présentent donc davantage d'animaux chers, car les artisans représentaient plus volontiers des scènes frappant l'imagination des gens qui aimaient participer aux grands sacrifices publics. Se basant sur une clause récurrente des lois sacrées, selon laquelle les particuliers pouvaient immoler les animaux qu'ils désiraient (à l'inverse des obligations relatées dans les calendriers publics), l'A. estime avec bon sens que les personnes agissant pour leur propre compte devaient être naturellement portées à sacrifier les animaux les moins chers: l'importance des porcins, dans les reliefs,

[éds], L'espace sacrificiel dans les civilisations méditerranéennes de l'Antiquité, Paris, 1991 [Publications de la Bibliothèque Salomon-Reinach, 5], p. 46-47).

23 Peut-être reprochera-t-on à l'auteur d'avoir négligé un autre type de sources (qu'il connaît bien, assurément, puisqu'il en parle aux p. 130-131) à savoir les restes d'ossements. Il en existe en contexte funéraire et cultuel : ne citons que ceux découverts par K. Kourouniotis, au début du siècle, sur le mont Lycée.

24 L'A. reprend le calendrier du dème de Thorikos, la loi de Nicomaque, le calendrier du dème d'Erchia, celui de la Tétrapole de Marathon et celui du genos des Salaminiens.

25 Peut-être les résultats varieraient-ils si l'on considérait non en général le type de bétail, mais la divinité elle-même, encore que les dieux n'étaient guère constants dans leurs exigences... L'écartement des chèvres (p. 171, n. 47) reste étonnant, quand on sait qu'elles étaient les victimes par excellence d'Artémis, au demeurant très rarement figurée en contexte sacrificiel. L'A. les trouve never very numerous, mais nous apprend qu'elles représentent : dans les calendriers attiques : $13 \%$ des victimes (alors que les bovins pris en compte totalisent 7,4\%); sur les vases: $9 \%$ (pour $13 \%$ et $12,2 \%$ aux moutons et aux porcs); dans les reliefs : $4 \%$. 
s'explique alors par le fait que le prix des porcelets était cinq fois moins élevé que celui d'un mouton et au minimum quinze fois moins que celui d'un bovin. On notera ici, en marge, qu'aucune figuration d'oiseau en contexte sacrificiel n'a été trouvée, alors qu'il est bien connu que certaines divinités, comme Asklépios, étaient grands demandeurs de gallinacés ${ }^{26}$.

Le prix et la valeur marchande des victimes ovines ont également pu être précisés; la différence de prix que les Grecs établissaient entre les moutons tient en effet au sexe de l'animal: la brebis était légèrement moins chère que le mouton appelé oís, lequel serait en fait un mâle châtré, donnant davantage de viande, qui lui-même était un peu moins dispendieux qu'un mâle non châtré,

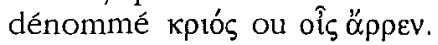

Le chapitre de conclusion prend fin sur une question importante (p. 186192): pourquoi les artisans choisirent-ils davantage de représenter la première phase, jamais la seconde, et plus rarement la troisième ? Ce débat ne pourra se résoudre en demeurant au niveau de l'interprétation première, et van Straten a préféré ne pas l'entamer, se contentant de donner quelques explications logiques mais non contraignantes : pour les reliefs (qui jamais ou presque ne figurent la phase post-sacrificielle, soit 1\%, et non assuré !), il avance que l'important, pour les particuliers, était de laisser une trace de leur sacrifice, et que seule la phase de l'arrivée à l'autel pouvait témoigner de la nature de l'animal représenté. Pour les vases, dont le caractère n'est pas souvent lié à cette notion de rappel d'un sacrifice particulier et dont le taux de figuration du rôtissage des $\sigma \pi \lambda \alpha v^{\prime} \chi \vee \alpha$ est assez important, il s'appuie sur le caractère fondamental de la communion avec les dieux et des présages favorables que ce moment permettait de constater. La sous-représentation du moment de la mise à mort enfin, contrastant avec la théorie de Burkert qui fait de l'Opfertötung la «fondamentale expérience vécue du sacré », est expliquée par le recours à un concept qui, pris au pied de la lettre, prend le contre-pied des hypothèses avancées par l'école de Vernant : soit, non par la thèse du «camouflage de la violence subversive », mais simplement par un manque total d'intérêt pour cet acte. Le choix des imagiers cependant, reste incompréhensible sur le fond, et pareilles hypothèses ne peuvent être prouvées ${ }^{27}$.

Le travail se clôt par une bibliographie de trente pages (p. 333-362), de nature exhaustive et qui comble un vide énorme, suivie par trois index (général, des sources littéraires et des inscriptions). Malheureusement, pour les catalogues, il manque un index des provenances, qui en eût rendu la consultation plus aisée, d'autant que le classement n'est pas chronologique mais « in broad iconograpbical groups and within these by region of manufacture ». Un second index reprenant, dans la description des pièces archéologiques, les principales carac-

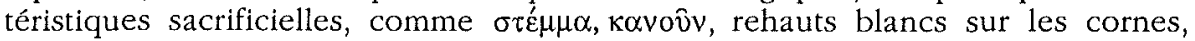
cordes entravant les pattes des victimes etc., eût été bien utile également. La

26 Par contre, je n'ai trouvé aucune allusion au sacrifice de thons, dont une image pourrait quand même passer pour sacrificielle (olpè à figures noires, Berlin, SM, F 1915), où figurent une table sacrificielle, un autel (?), un couteau sacrificiel et un vase à saigner : même si la scène s'avérait non religieuse, il n'en reste pas moins qu'elle est étonnamment proche du sacrifice! Il est vrai que nos informations à propos des sacrifices de poissons sont dérisoires: to say notbing of fish, dit W. BURKERT (Greek Religion. Archaic and Classical, trad. J. Raffan, Cambridge [Mass.], 1985, p. 55 et n. 4, avec [maigres] références).

27 Dans son introduction d'ailleurs (p. 3-5), l'A. appelait lui-même à la prudence dans l'interprétation de cette face de la documentation : il donnait l'exemple de l'inexistence de représentations de sacrifices purificatoires (à l'exception de quelques scènes mythiques), alors qu'à Athènes il devait s'en pratiquer quotidiennement. 
richesse de l'illustration est à souligner (168 photographies noir et blanc), mais en même temps on regrettera que la Maison Brill n'en ait pas profité pour publier tout le corpus quitte, en certains cas, à recourir au dessin au trait.

En conclusion, le travail de van Straten est un merveilleux aboutissement, clair, net, concret, et qui promet de riches études subséquentes dans un futur proche.

\section{Le sacrifice animal à la lumière du sacrifice humain}

F. van Straten a eu le mérite aussi de cataloguer les scènes de sacrifices humains ${ }^{28}$ mais, fidèle à sa ligne de conduite du «What you see is what it is », il a considéré ces illustrations dans ce qu'elles pouvaient nous apprendre sur la réalisation concrète du sacrifice, sans aborder leur signification symbolique et ses implications ${ }^{29}$. Je voudrais, pour terminer, lancer quelques pistes à propos des relations entre sacrifice animal et sacrifice humain, dont l'étude conjuguée apporte tant à la connaissance du premier que du second, tout en faisant progresser la compréhension du fonctionnement de l'esprit hellénique.

L'inexistence du sacrifice humain en Grèce et le rejet unanime de celui-ci par les Grecs $^{30}$ ne doivent pas faire oublier que ces mêmes Grecs étaient confrontés dans leur mythologie à plus de soixante cas de sacrifices humains, certains accomplis par leurs ancêtres et dont l'authenticité ne fut que rarement mise en doute $^{31}$. De plus, le sacrifice humain acquit en Grèce un caractère profondément ambigu : en ce que l'homme y apparaissait comme la victime sacrifiée, le

28 J'en ajoute quelques autres qui, sans être toujours évidentes, sont susceptibles d'apporter quelques précisions (à moins que je ne les aie manquées dans les catalogues). Macarie (?) : cratère à volutes du Groupe du Peintre de l'Iliupersis, Bari, $M N, 3648$ (ca 360 av. J.-C.; LIMC, Makaria, $\mathrm{n}^{\circ}$ 3); cratère en cloche campanien du Peintre de Cavaino, Schwerin, NM, inv. 719 (ca 340-330 av. J.-C.; LIMC, Makaria, n 2). Médéides : cratère en coupe lucanien d'un peintre apparenté au Peintre de Policoro, Fort Worth, Kimbell Art Mus., coll. Hunt ( $c a 400$ av. J.-C.; LIMC, Medeia, $\mathrm{n}^{\circ}$ 36); amphore campanienne du Peintre du $B M$ F 223, Paris, Cabinet des Médailles, 876 (ca 330 av. J.-C.; LIMC, Medeia, $\mathrm{n}^{\circ} 30$ ); cratère à volutes apulien du Peintre des Enfers, Munich, Antikensammlung, 3296 ( $c a 330$ av. J.-C.; LIMC, Medeia, $\mathrm{n}^{\circ}$ 29); amphore (fr.) apulienne d'un peintre apparenté au Peintre de Darius, Halle, Universität, 215 (ca 340-320 av. J.-C.; LIMC, Medeia, n²8). Oreste : amphore apulienne perdue (LIMC, Iphigeneia, $\mathrm{n}^{\circ} 20$ ); cratère du Peintre de Baltimore, Saint-Petersbourg, Ermitage, B 1715A (ca 330-320 av. J.-C.; LIMC, Ipbigeneia, $\mathrm{n}^{\circ} 23$ ); cratère en coupe apulien du Peintre de l'Iphigénie de Moscou, Moscou, Mus. Pouchkine, 504 ( $c a$ 345-340 av. J.-C.; LIMC, Ipbigeneia, $\mathrm{n}^{\circ} 22$ ). Phrixos : amphore attique du Peintre de Munich 2335, Naples, $M N$, SA 270 (ca 450-425 av. J.-C.; LIMC, Phrixos, $\mathrm{n}^{\circ} 2=$ Inô, $\mathrm{n}^{\circ} 13$ ). Polyxène : hydrie campanienne du Peintre de Cavaino, Naples, $M N$ ( ca 340-330 av. J.-C.; LIMC, Polyxena, $\mathrm{n}^{\circ}$ 39); amphore paestane du Peintre de Naples 1778, Naples, MN, H 1779 (ca 315 av. J.-C.; IIMC, Polyxena, n 40).

29 Encore n'a-t-il examiné que le moment de la mise à mort, et non les attestations de procession ou les actes préliminaires dans les sacrifices humains (p. 113-114); d'intéressantes comparaisons sont pourtant possibles, par exemple entre les V 384, ill. 43 et V 397, ill. 117, issus du même atelier et figurant le même moment, ou presque, respectivement dans les deux types de sacrifice.

30 Voir P. BonNechere, Le sacrifice bumain en Grèce ancienne, Athènes-Liège, 1994 (Kernos, suppl. 3); A. Henrichs, Human Sacrifice in Greek Religion. Three Case Studies, in Le sacrifice dans l'Antiquité (cité n. 3), p. 195-235; M. Halm-Tisserant, Cannibalisme et immortalité. L'enfant dans le cbaudron en Grèce ancienne, Paris, 1993 (Vérité des mythes); D.D. Hugres, Human Sacrifice in Ancient Greece, Londres-New York, 1991.

31 BonNechere, Le sacrifice bumain en Grèce ancienne (cité n. 30), p. 249. 
sacrifice humain était l'inverse du sacrifice animal et en tant que tel suscitait une profonde répulsion. Mais en même temps, parce que la victime humaine était exceptionnelle et supérieure à l'animal, cet acte atroce touchait paradoxalement à la perfection sacrificielle ${ }^{32}$. Dans ce contexte, il est instructif de s'interroger sur la façon dont les Grecs imaginaient le déroulement du sacrifice humain, qui apparaissait comme un sous-ensemble de la sphère sacrificielle, aux côtés des offrandes animales et des offrandes non sanglantes. Il faut, en d'autres termes, tenter de voir, en considérant aussi bien les sources littéraires que les sources iconographiques ${ }^{33}$, en quoi et dans quelle mesure celui-ci se distinguait du sacrifice animal. Grâce à l'ouvrage de van Straten, des comparaisons sûres, sans aucun a priori méthodologique, sont désormais réalisables.

Le sacrifice humain, tout imaginaire soit-il, est segmentable selon la même succession d'actes que le sacrifice animal ${ }^{34}$, dont il possède d'ailleurs le caractère foncièrement collectif ${ }^{35}$ : procession et habillement des participants, tenue et nature des victimes, actes préliminaires (circumambulation, prière, amenée de l'arme et des grains d'orge, silence rituel, etc.), consécration, approbation par la victime humaine de son propre sacrifice, empoignement de la victime et mise à mort. La phase post-sactificielle est très rarement évoquée, en raison des substitutions animales et des nombreux sacrifices avortés, mais il est des cas qui y font référence, une fois peut-être avec prise des présages ${ }^{36}$ et à plusieurs reprises avec

32 Ibid., p. 311-314.

33 Les documents figurés relatifs aux traditions grecques de sacrifice humain, dans le monde gréco-romain, sont aux alentours de cinq cents, et leur apport, parfois providentiel, a toujours été ignoré, à l'exception de quelques comparaisons isolées. Ils offrent cependant aux textes une contrepartie indispensable, et même si leur nombre n'est pas proportionnel à l'intérêt qu'on peut en retirer, une bonne centaine peut être valablement interrogée. Après un dépouillement complet, il s'avère que seul un nombre restreint de légendes a été illustré, à savoir les sacrifices d'Iphigénie à Aulis, d'Oreste et Pylade en Tauride, de Polyxène sur la tombe d'Achille, d'Héraclès par Busiris, des enfants de Médée, et enfin des prisonniers troyens au bûcher de Patrocle. Il s'agit néanmoins des cas les plus célèbres en Grèce, racontés par Euripide pour les premiers et par Homère pour le dernier. Quelques autres cas pourraient avoir été figurés, mais dont l'identification ne fait pas l'unanimité; citons Macarie (voir M. ScHMiDT, Makaria, in AK, 13 [1970], p. 71-73), ou une Érechtéide durant la guerre contre Eumolpos, sur la frise du Parthénon (voir J. Conneliy, Partbenon and Parthenoi. A Mytbological Interpretation of the Partbenon Frieze, in $A J A, 100$ [1996], p. 53-80). Enfin, d'autres cas annexes peuvent être pris en considération en ce qu'ils comportent des similitudes évidentes avec les cas de sacrifice humain : Andromède et Hésione offertes au monstre, Actéon déchiqueté par ses chiens, le fils de Lycurgue abattu par son père, ou Orphée et Penthée écartelés par les Ménades. La plupart des illustrations sont désormais publiées dans le LIMC.

34 Il est nécessaire d'envisager tous les types de sacrifices « chthoniens et olympiens ", pour les regrouper sous une appellation facile; en effet, le sacrifice humain n'étant qu'une réalité imaginaire, il échappe à toute tentative de classement, déjà difficile pour les sacrifices animaux : l'immolation d'Iphigénie se transforme au gré des sources (Euripide et Eschyle par exemple), dans un rituel qui ressemble à une $\theta$ voí $\alpha$ mais sans manipulation des exta, sans festin, avec une victime nommée $\sigma \varphi \alpha ́ \gamma$ rov et offerte à Artémis alors qu'elle va épouser Hadès, etc.

35 Que ce soit en fonction du groupe sacrifiant ou de la représentativité du sacrificateur (rois, généraux...). J'ai consacré un article à ce sujet, qui devrait paraître sous peu dans Pboenix.

36 Si l'on considère en ce sens l'égorgement de Macarie dans les Héraclides d'Euripide (819-822), à la lumière de van Straten, Hierà kalá, p. 156-157 et W.K. PritcheTt, The Greek State at War, 3: Religion, Berkeley-Los Angeles, 1979, p. 48 sq. 
grillade des entrailles ${ }^{37}$. Le traitement des cadavres, manducation ou inhumation /crémation sans repas sacrificiel, est assez rarement décrit.

Sans vouloir anticiper sur une étude approfondie que je tente de mener à bien, j'en donnerai quelques exemples. Ainsi la $\pi 0 \mu \pi \eta ́$ des victimes humaines estelle bien attestée dans les textes ${ }^{38}$, mais elle ne s'organise pas de façon comparable à celle des sacrifices animaux; on n'y distingue aucun transport des objets nécessaires au sacrifice, tandis que l'iconographie révèle, par exemple, dans la tenue vestimentaire des acteurs, l'absence de couronnes ${ }^{39}$, dont la signification semble liée à l'inversion du rite traditionnel, à l'exception logique des étrangers sacrifiants, comme Busiris ou Thoas ${ }^{40}$. Lors de la procession d'un sacrifice humain, on conduit la victime à l'autel parce qu'il faut bien l'y mener, mais tout caractère de liesse a été scrupuleusement effacé, parce que l'acte posé, en des circonstances de renversement, bien qu'il soit exigé par la divinité, est aux antipodes du sacrifice qui régit le cosmos dans les conditions normales. La procession avec victime humaine, essentiellement féminine, nourrit par ailleurs tellement de rapports et de parentés avec les processions funéraires et nuptiales, qu'il apparaît évident que chacune des trois devait susciter automatiquement, dans un esprit grec, une pensée relative aux deux autres ${ }^{41}$.

L'autel est l'élément par excellence du paysage sacrificiel, et il semble jouer par métonymie, qu'elle soit textuelle ou visuelle : «mener la victime à l'autel » est en effet un euphémisme pour "sacrifier », tandis que les figurations montrent pour la plupart ce dernier dans une position où il attire tous les regards. Souvent les traces de sang sur l'autel prédisent l'avenir sinistre de la scène de procession animale ${ }^{42}$. Dans le cas de victimes humaines, ces traces de sang acquièrent un sens plus profond. En Égypte, Busiris passe pour immoler tous les étrangers qui mettent le pied dans le pays, et l'autel rougi de sang sur les vases qui figurent Héraclès sur le point d'être sacrifié renvoie évidemment aux autres sacrifices humains qui s'y sont déroulés auparavant et qui devraient s'y dérouler à l'avenir, conformément à l'image mentale que les Grecs se faisaient des usages sacrificiels

$37 \mathrm{Y}$ compris parfois la phase de l'ingestion des chairs des victimes (Pélops, fils de Lycaon), sans parler des $\sigma \pi \alpha \rho \alpha \gamma \mu$ oí dionysiaques inversifs : voir en dernier lieu HaLMTisserant, Cannibalisme et immortalité (citée n. 29), passim.

38 Par ex. : Aél. Arist., I, 87; Hdt., II, 45; VII, 197; Paus., VII, 19, 8 et 20, 1; VII, 21, 4; Plut., Thémist., 13, 5; schol. ad Aristoph., Nuées, $257 \mathrm{~b}$ et d, etc. Voir mon article $L a$ pompè sacrificielle des victimes bumaines en Grèce (cité n. 7).

39 Par ex. pour Iphigénie: oinochoé attique du Peintre de Schuwalow, Kiel, Universität, B 538 (ca $430-420$ av. J.-C.; LIMC, Ipbigeneia, $\mathrm{n}^{\circ} 1$ ); cratère à volutes apulien d'un peintre apparenté au Peintre de l'Iliupersis, Londres, BM, F 159 (ca 370-355 av. J.-C.; LIMC, Ipbigeneia, $\mathrm{n}^{\circ} 11$ ), etc.

40 Par ex. pour Busiris : stamnos attique du Peintre de Pan, Leipzig, Universität, T 651538 ( ca 470 av. J.-C.; LIMC, Bousiris, $\mathrm{n}^{\circ}$ 21); cratère à colonnettes attique (fr.), Boston, MFA, 10.188538 (ca 470-460 av. J,-C,; LIMC, Bousiris, $\mathrm{n}^{\circ} 24$ ); pélikè attique du Peintre des Éthiopiens, Paris, Cabinet des Médailles, 393 (ca 460 av. J.-C.; LIMC, Bousiris, $\mathrm{n}^{\circ} 1$ [= Aitbiopes, $\left.\mathrm{n}^{\circ} 181\right)$ etc.

41 Voir par ex. I. Jenkins, Is there a Life after Marriage? A Study of the Abduction Motif in Vase-Paintings of the Atbenian Wedding Ceremony, in BICS, 30 (1983), p. 137-145: l'A. met en parallèle le lécythe de Douris (Palerme, MR, NI 1896; ca 470 av. J.-C.; LIMC, Ipbigeneia, $\mathrm{n}^{\circ}$ 3) et une pyxide du Peintre Splanchnopt (Londres, BM, D 11; ca $470-460$ av. J.-C.; Jenkins [cité ci-dessus], pl. 18a) qui présentent tous deux une procession, sacrificielle dans le premier cas, nuptiale dans le second, et dont les affinités sont évidentes. Voir aussi M. Alexiou, The Ritual Lament in Greek Tradition, Cambridge, 1974, passim.

42 Voir essentiellement DuRAND, Images pour un autel (cité n. 22), p. 45-55. Voir par ex. van Straten, Hierà kalá, V 132, V 147, ill. 110, V $266 .$. 
busiriens ${ }^{43}$. Mais lorsqu'un peintre apparenté au Peintre de l'Iliupersis figure, dans le sacrifice d'Iphigénie ${ }^{44}$, un autel maculé de sang, la signification du détail devient plus complexe encore : il ne s'agit pas tant de manifester ici une

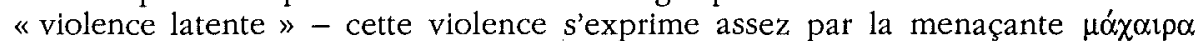
qu'Agamemnon pointe vers sa fille - que de brouiller les normes, ou d'accuser davantage encore les limites entre sacrifice humain et sacrifice animal, les marques de sang sur le $\beta \omega \mu$ ó $\varsigma$ résultant du bon usage du sacrifice, en contraste avec l'usage anormal qui va s'y accomplir. En cela, ces traînées rouges appartiennent à ce groupe d'idées qui focalisent l'attention du spectateur sur la confusion entre victime animale et victime humaine. L'allusion est plus fine encore sur une pélikè lucanienne d'un peintre du Groupe de Policoro ${ }^{45}$, qui montre, réfugiés sur l'autel de Marathon, les Héraclides et Iolaos subissant l'impertinence du héraut argien. Le sang sur l'autel trahit la violence animale mais y est également prémonitoire du sacrifice de Macarie, alors que celle-ci est absente de l'image, conformément à la tragédie d'Euripide où elle est terrée à l'intérieur du temple.

La phase de l'égorgement est de loin la plus problématique. Abordons ce point, en matière de sacrifices humains, avec le problème de l'empoignement des victimes à l'autel, tout en gardant présente à l'esprit la devise de van Straten. Sans doute avons-nous des images, corroborées par certains textes, qui montrent une jeune fille saisie ${ }^{46}$, soulevée comme un taureau sur l'amphore de Viterbo ${ }^{47}$, pour être présentée au couteau du sacrificateur.

Sur l'amphore de Viterbo, la violence est extériorisée, non seulement par la mise en évidence de l'arme mais encore par celle des musculatures tendues, particulièrement pour deux personnages : le premier qui tient le bœuf par la queue, le second qui se laisse pendre de tout son poids sur une corde qui musèle un animal totalement dominé et violenté, aux antipodes de la libre volonté. Estce à dire que la victime n'a pas accepté son propre sacrifice, comme la tradition le voudrait? Certainement pas : l'imagier a pu figurer ici l'étape postérieure à l'arrivée devant l'autel traditionnellement représentée, comprenant l'acceptation animale, car on a beau cacher la violence, - que ce soit à l'animal ou au citoyen, - il arrive un moment où elle se manifeste. Si une part importante de l'iconographie du sacrifice animal a figé l'instant où le sacrifiant va asperger l'animal pour obtenir son consentement, il va de soi qu'une fois cette acceptation obtenue, la bête se débattra au moment où on la soulèvera et plus encore au moment de la mise à mort ${ }^{48}$, mais cette violence est désormais tolérable car ses acteurs en sont déchargés de la culpabilité.

43 Par ex. : hydrie attique du Peintre de Troilos, Munich, Antikensammlung, 2428 (ca 490 av. J.-C.; LIMC, Bousiris, $\mathrm{n}^{\circ}$ 15); pékikè attique du Peintre de Pan, Athènes, MN, 9683 (ca 490-470 av. J.-C.; LIMC, Bousiris, n²0).

44 Cité n. 39.

45 Tarente, $M N$ ( $c a 400$ av. J.-C.; A.D. Trendall, T.B.L. WeBster, Illustrations of Greek Drama, Londres, 1971, p. 86 [III.3, 20]).

46 Par ex. pour Polyxène : amphore tyrrhénienne attique du Peintre de Timiadès, Londres, BM, 97.7-27.2 (ca 575-550 av. J.-C.; LLMC, Polyxena, $\mathrm{n}^{\circ} 26$ ).

47 VAN STRATEN, Hierà kalá, V 141, ill. 115 ( ca 550 av. J.-C.); publication approfondie chez G. Barbieri, J.-L. Durand, Con il bue a spalla, in $B d A, 70$ (1985), p. 1-16.

48 Sur nombre de vases (J.-L. DuRAND, Sacrifice et labour en Grèce ancienne. Essai d'antbropologie religieuse, Paris-Rome, 1986, fig. 58-61), par exemple, on voit un sacrificateur porter d'une main un cochonnet par une patte et de l'autre le kovôvv : rien n'empêche de considérer que le moment figuré est celui qui suit l'acceptation de l'animal et qui précède l'immolation. Même chose pour le bélier transporté à l'autel sur un cratère 
En cela, l'amphore tyrrhénienne ${ }^{49}$ qui montre Polyxène égorgée comme le taureau sur la tombe d'Achille, est à la fois proche et radicalement différente : la jeune fille subit certes la même violence que l'animal, à cette différence près qu'avant Euripide, on peut gager avec une presque totale certitude que jamais il n'avait été question pour elle d'accepter sa mise à mort. La violence est donc extériorisée de même, si ce n'est qu'elle se manifeste dans l'inversion des valeurs sacrificielles ${ }^{50}$. On remarquera par ailleurs que, dans l'Hécube d'Euripide, ces deux notions réapparaissent de façon solidaire ${ }^{51}$ : les éphèbes choisis parmi l'armée achéenne sont prêts à saisir la jeune fille, mais le discours de Polyxène, qui justifie l'acceptation de sa mise à mort, fait en sorte que cette saisie brutale n'est plus nécessaire, la violence se limitant alors au coup fatal. La Polyxène d'Euripide est donc différente de l'animal, car son acceptation du sacrifice ne relève pas d'une comédie de l'innocence mais d'une pleine compréhension des faits : la similitude de représentation ne trahit donc pas la similitude de signification.

Sous des dehors très semblables parfois se cachent de subtiles nuances qui définissent à quel point les Grecs, dans l'ensemble, établissaient une distinction, du point de vue de l'imaginaire s'entend, entre sacrifice d'un être humain et sacrifice d'un être animal.

apulien représentant le sacrifice précédant la course mythique d'Oinomaos et Pélops (van Straten, Hierà kalá, V 409, St Petersbourg, 4323).

49 Citée n. 15.

50 Selon un schéma très proche de celui du sacrifice d'Iphigénie dans l'Agamemnon d'ESCHYLE (192-247).

51 Eur., Héc., 521-582. 